\title{
Critical Parameters of Gas Cavities in Dielectric Liquids Stressed with High Electric Fields
}

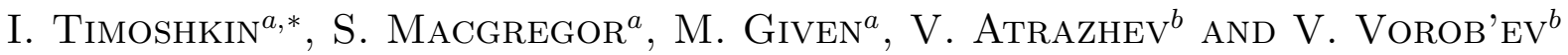 \\ ${ }^{a}$ Department of Electronic and Electrical Engineering, University of Strathclyde \\ 204 George Street, Glasgow, G1 1XW, UK \\ ${ }^{b}$ Joint Institute for High Temperatures, Russian Academy of Sciences \\ 12/14 Izhorskaya St., Moscow, 127412, Russia
}

\begin{abstract}
Electric field stimulates formation of gas-filled cavities on sharp electrodes in insulating liquids which may initiate the dielectric breakdown of these liquids. Using an analytical model based on electrical and thermodynamic properties of fluids, the critical dimensions of gas-filled bubbles in ester fluid and transformer oils stressed with an impulse electric field were calculated. The results obtained in this paper could be important for the optimisation of the application of these liquids in high voltage and pulsed power systems.
\end{abstract}

PACS numbers: $52.80 . \mathrm{Wq}, 77.22 . \mathrm{Jp}$

\section{Introduction}

Insulating liquids including naphthenic mineral oils and synthetic ester fluids have found applications as dielectric materials in a wide range of pulsed power and high voltage systems. These dielectric fluids are used in different components of pulsed power systems, such as pulse forming lines, energy storage components, capacitors and switches. Analysis of the physical processes in the liquids when stressed with a high pulsed electric field is essential for further understanding of the breakdown mechanisms and could help in the optimisation of existing and in the development of new applications of the liquid insulators.

This paper presents an analytical analysis of the formation of pre-breakdown micro-cavities in an insulating non-conductive liquid stressed with a high electric field. Development of such gas-filled cavities is considered as one of the possible mechanisms which result in the dielectric breakdown of liquids [1]. The application of a sufficiently high voltage pulse, with a sub-microsecond rise time, to a sharp (point) electrode immersed in the dielectric liquid may result in the formation of a vapour-filled cavity on the electrode tip. If this cavity exceeds a critical size it starts to grow and may be considered as an initial stage in the dielectric breakdown of the insulating liquid. It is assumed that this cavity (bubble) on the electrode tip appears due to small density fluctuations existing in the fluid. These fluctuations are short-lived in the liquid and they disappear without any changes in the thermodynamic state of the liquid.

\footnotetext{
* corresponding author; e-mail: igor.timoshkin@eee.strath.ac.uk
}

However, the application of the external electric field to the liquid changes this situation. As has been shown in $[3,4]$, the external electric field promotes the formation of the bubbles through the change in the thermodynamical properties of the liquid and its phase equilibrium conditions. The electrostrictional tensile stress exerted on the liquid in the external field results in the "rupture" of the liquid and the formation of gas cavities. As soon as this initial cavity is formed, an additional electrostatic surface force appears across the cavity/liquid interface. This force is proportional to the square of the field magnitude, $E^{2}$, similar to the electrostriction force, and also to the difference between the dielectric permittivities of the liquid and the media inside the cavity [2].

After its formation, the bubbles with or above a critical radius start to grow due to intensified vaporisation of the liquid from the bubble/liquid interface. This process can lead to bubbles of sufficient size that dielectric breakdown of vapour inside the bubble occurs. Analytical evaluation of the critical radius of the bubble and the critical magnitude of the external field which can trigger the formation of this bubble may help in determining the potential applicability of insulating liquids in different pulsed power systems. The dynamics of the behaviour of the bubbles are discussed in Ref. [5].

\section{Analytical analysis of the critical parameters of the bubbles}

In the present analysis the point-plane electrode configuration is considered. It is assumed that the liquid is non-conductive and the duration of the voltage impulse is sufficiently short, so the Joule heating of the insulating liquid is absent. As was discussed in Introduction, a strong electric field changes the thermodynamical 
properties and the phase equilibrium conditions for the liquid, generating a tensile stress and a surface electrostatic force. Sufficiently strong electric field can potentially transfer the liquid into its metastable overheated state and trigger the formation and development of a gas bubble. The electric field has its maximum on the tip of the point electrode. Hence, the electrode's tip is the most probable area in the liquid where a bubble can be formed. The work $A_{c}(E)$ required to form the bubble with the critical size $R_{c}(E)$, may be written as $[3,4,6,7]$ :

$$
\begin{aligned}
& A_{c}(E)=A_{c}(0)(1-5 x) /(1-x)^{3}, \\
& R_{c}(E)=R_{c}(0) /(1-x),
\end{aligned}
$$

where $R_{c}(0)=2 \sigma /|\Delta p|$ is the radius of the critical sized bubble when the field is absent, and $\sigma$ is the surface tension. In Eqs. (1), (2) $x$ is defined as $x=\Delta F / \Delta p$. It may be assumed that the difference between the hydrostatic pressure in the liquid, $p_{0}$, and the saturated vapour pressure inside the bubble, $p_{\text {sat }}, \Delta p=p_{0}-p_{\text {sat }}$, is equal to the hydrostatic pressure, $p_{0}$, since $p_{0} \gg p_{\text {sat }}$. The quantity $\Delta F$ is the surface electrostatic force which is associated with the difference between permittivities of the liquid, $\varepsilon_{2}$, and the vapour, $\varepsilon_{1}$

$$
\Delta F=\frac{\varepsilon_{1}-\varepsilon_{2}}{8 \pi}\left(E_{t}^{2}+E_{n}^{2} \frac{\varepsilon_{1}}{\varepsilon_{2}}\right),
$$

where $E_{t}$ and $E_{n}$ are the normal and tangential components of the field at the bubble/liquid interface [4].

The electric field on the electrode tip can be modelled at one extreme by the field generated by an ideal charged point. Another limiting situation is the uniform electric field between two parallel-plane electrodes. These two limiting cases require different values of the parameter $x$ in Eq. (1), which have been obtained in $[3,4,6,7]$ and are used here for analysis of the electric field on the electrode's tip.

As it follows from Eq. (1), the work $A_{c}(E)$ is equal to zero if $\Delta F / p_{\text {liq }}=1 / 5$. This condition determines the minimal (critical) magnitude of the electric field which is necessary to form a critical sized bubble. In the case of the strongly non-uniform electric field, this critical field, $E_{c c}$, is

$$
E_{c c}=\frac{0.67}{\left(1-1 / \varepsilon_{2}\right)^{0.5}}[\mathrm{MV} / \mathrm{cm}] .
$$

In the case of the uniform electric field, the critical magnitude, $E_{c u}$, can be expressed as

$$
E_{c u}=0.77\left[\left(1+\varepsilon_{2} / 2\right) /\left(1-1 / \varepsilon_{1}\right)\right]^{0.5}[\mathrm{MV} / \mathrm{cm}] .
$$

The vapor bubble will not be formed if the field magnitude is less than the critical value. As soon as the field's magnitude becomes equal to or greater than the critical value, the bubble forms on the tip of the electrode. However, the homogeneous liquid dielectric does not exist when the field magnitude is greater than $E_{c}$.

\section{Results and discussion}

Using the analytical expressions discussed in Sect. 2, the critical radius of the bubble, $R_{c}$, and the critical field magnitudes, $E_{c c}$, and $E_{c u}$, have been calculated for two transformer insulating oils (Diala AX and Voltesso 35), the synthetic ester insulating liquid (Midel 7131) and the silicone lubrication liquid (Dow Corning 710). Calculated critical parameters together with the physical properties of these liquids used in the present analysis are given in Table.

\section{TABLE}

Physical properties of liquids and calculated critical parameters.

\begin{tabular}{l|c|c|c|c|c|c}
\hline \hline \multicolumn{1}{c|}{ Liquid } & $\varepsilon$ & $\begin{array}{c}\text { Vapour } \\
\text { pressure } \\
{[\mathrm{mmHg}]}\end{array}$ & $\begin{array}{c}\sigma \\
{[\mathrm{mN} / \mathrm{m}]}\end{array}$ & $\begin{array}{c}E_{c c} \\
{[\mathrm{MV} / \mathrm{cm}]}\end{array}$ & $\begin{array}{c}E_{c u} \\
{[\mathrm{MV} / \mathrm{cm}]}\end{array}$ & $\begin{array}{c}R_{c} \\
{[\mu \mathrm{m}]}\end{array}$ \\
\hline Diala AX (Shell) & 2.3 & $3.8 \times 10^{-3}$ & $\sim 30$ & 0.89 & 1.14 & 0.72 \\
Midel 7131 & 3.2 & $7.5 \times 10^{-8}$ & $\sim 25$ & 0.81 & 1.0 & 0.60 \\
Voltesso 35 & 2.2 & $3.0 \times 10^{-3}$ & 29.9 & 0.91 & 1.16 & 0.71 \\
Dow Corning 710 & 2.3 & negligible & 28.5 & 0.89 & 1.14 & 0.68
\end{tabular}

According to this analysis, the Midel 7131 and Dow Corning 710 liquids have the lowest magnitude of the critical field and the smallest critical radius of the electrically stimulated gas bubbles. This suggests that the dielectric breakdown strength of these two liquids should be lower than the breakdown strength of the insulating oils considered here which is confirmed by the experimental data presented in report [8] and in paper [9]. According to these results the impulse breakdown strength of the Diala D oil is higher than the impulse strength of the Midel 7131 liquid. However, when stressed with AC voltage, the insulating liquids may behave differently. For example, paper [10] reports a lower breakdown strength for the mineral oil as compared with the ester liquid under $50 \mathrm{~Hz}$ high voltage stress which can be attributed to different breakdown mechanisms under the impulse and AC high voltage stress. 


\section{Acknowledgments}

This work has been supported by the Royal Society through the award of the Joint International Grant in 2007. V.A. and V.V. are grateful to the University of Strathclyde for hospitality during their stay in Glasgow.

\section{References}

[1] A. Beroual, J. Appl. Phys. 73, 4528 (1993).

[2] L. Landau, E. Lifshitz, Electrodynamics of Continuous Media, Pergamon, Oxford 1984.

[3] V. Vorob'ev, S. Malyshenko, Zh. Eksp. Teor. Fiz. 120, 863 (2001).

[4] V. Vorob'ev, S. Malyshenko, A. Petrin, J. Phys. D 35, 257 (2002).
[5] T. Aka-Ngnui, A. Beroual, J. Phys. D: Appl. Phys. 34, 1408 (2001).

[6] V. Vorob'ev, S. Malyshenko, Exp. Therm. Fluid Sci. 26, 833 (2002).

[7] V. Vorob'ev, S. Malyshenko, Appl. Phys. Lett. 60, 371 (2002).

[8] S. MacGregor, I. Timoshkin, M. Given, Study of the dielectric properties of Diala D and Midel 7131 insulating fluids, Internal report, University of Strathclyde, 2007.

[9] P. Leask, Acta Phys. Pol. A 115, 998 (2009).

[10] F.C. Perrier, A. Beroual, J.-L. Bessede, IEEE Int. Symp. Electrical Insulation, Indianapolis, USA 2004 , p. 237. 